\title{
An Improved Technique for Sorting Developmental Stages and Assessing Egg Viability of Globodera pallida using High-Throughput Complex Object Parametric Analyzer and Sorter
}

\begin{abstract}
Abolfazl Hajihassani ${ }^{\dagger}$ and Louise-Marie Dandurand, Department of Entomology, Plant Pathology, and Nematology, University of Idaho,
\end{abstract} Moscow 83844

\begin{abstract}
The Complex Object Parametric Analyzer and Sorter (COPAS) is a large particle flow cytometer designed for analyzing, sorting, and dispensing objects of varying sizes. We explored the potential of using this instrument to analyze and sort various developmental stages and egg viability of Globodera pallida. Cysts were successfully examined and sorted from debris by optimizing side-scatter and red-fluorescence parameters on the COPAS. We were able to separate eggs and second-stage juveniles from samples of mixed population using extinction and time of flight. Separation of live and dead eggs was examined following staining eggs with SYTOX Green and application of time of flight and green

peak height. Data were compared with a commonly used viability assay by which eggs were stained with Meldola's Blue and examined by a microscope. COPAS proved to be effective in assessing viability by detecting two separate gates: live eggs having green fluorescence peaks $<190$ and dead eggs with the peaks $>190$. The application of COPAS in combination with SYTOX Green detected a greater number of live eggs than the Meldola's assay, suggesting that SYTOX Green provided an overestimate of live eggs. COPAS noticeably increased the accuracy and reduced the time required for screening and analyzing nematode populations.
\end{abstract}

Estimation of plant-parasitic nematode populations is of great importance for accurate examination of host plant responses to nematode infection in most applied and fundamental nematology research. Additionally, accurate estimation of nematode population density from soil or plant samples is a major step in assessing potential crop damage caused by the nematodes and could consequently result in appropriate decision making for management tactics as well as for use in regulatory application. For cyst-forming nematodes, such as Globodera spp. and Heterodera spp., however, the estimation of population densities are more challenging and complex because the number of cysts is not the only measure for determination of a nematode population but rather the number of encysted eggs and an assessment of their viability.

The potato cyst nematode (PCN), Globodera pallida (Stone 1973) Behrens 1975, is an economically important pest worldwide with potential to threaten global food security and is a regulated pest in many countries throughout the world (Evans and Stone 1977; OEPP/EPPO 2013; Turner and Evans 1998). For the United States, G. pallida is a quarantine pest for which there is zero tolerance. The presence of $G$. pallida in Idaho was viewed with extreme alarm by other states and countries that import Idaho potato. Prior to implementation of the current quarantine measures, import of Idaho fresh potato products and nursery stock was banned by some of Idaho's most important trading partners, including Canada, Mexico, and Japan. Consequently, eradication of $G$. pallida is a top priority for the Idaho potato industry, including the Idaho Potato Commission, Idaho State Department of Agriculture, and the United States Department of Agriculture-Animal and Plant Health Inspection Service (USDA-APHIS). A critical component of this work has been undertaken by USDA-APHIS through intensive sampling and fumigation of infested fields. Viability

Current affiliation of A. Hajihassani: Department of Plant Pathology, University of Georgia, Tifton, GA 31794.

${ }^{\dagger}$ Corresponding author: A. Hajihassani; E-mail address:

abolfazl.hajihassani@uga.edu

Accepted for publication 10 April 2018.

C) 2018 The American Phytopathological Society assessments are made after fumigation to evaluate the progress of the eradication program (Anonymous 2014).

No efficient method, except time-consuming and labor-intensive manual processing and microscopic observation, is currently available for sorting cyst populations of PCN. In addition, determination of whether a PCN population is viable (live) or nonviable (dead) is essential, considering the fact that detection of only one cyst in the soil can lead to a potential threat to crop production systems (Back et al. 2004; Kroese et al. 2011) or regulatory action. A number of procedures have been used to find out which egg individuals are alive and which are not; however, variable outcomes have been reported for some of these techniques. Because distinguishing between live and dead eggs by visual examination under a microscope is not always possible, several conventional techniques such as egg staining with fluorescent and nonfluorescent stains (Ogiga and Estey 1974; OEPP/EPPO 2013), egg hatching in response to host and nonhost plant root diffuses (Devine et al. 1996), and a combination of egg staining and hatching (Kroese et al. 2011) bioassays have been used to determine the viability of a cyst nematode population. Many nonfluorescent stains such as Meldola's Blue, eosin Y, and Nile Blue A (Ogiga and Estey 1974; Shepherd 1962) have been extensively used for testing the viability of Meloidogyne incognita, Heterodera glycines, G. rostochiensis, and G. pallida (Kroese et al. 2011; Meyer et al. 1988). These staining assays provide satisfactory assessment of the viability but they are time-consuming and tedious and are subjected to enumerating errors that make them unreliable for routine usage (Back et al. 2004; Beniers et al. 2014).

The potential use of flow cytometry in combination with fluorescent viability indicators such as SYBR Green and SYTOX Green has been reported as a suitable technique for rapid and precise quantification of live and dead bacteria (Lebaron et al. 1998; Porter et al. 1995). More recently, a trehalose-based technique (Beniers et al. 2014; Ebrahimi et al. 2015; van den Elsen et al. 2012) has been established to estimate and quantify the viability of PCN. Additionally, a few RNA-based techniques have been developed for testing the viability of nematode eggs. A reverse-transcription polymerase chain reaction (PCR) method on the basis of the identifying mRNA from the glyceraldehyde-3-phosphate dehydrogenase 1 ( $g p d l$ ) gene has been developed for assessing the viability of PCN (Kaemmerer 2012). Christoforou et al. (2014) established a quantitative PCR-based technique combined with propidium monoazide stain for separating live and dead eggs of G. pallida and G. rostochiensis. These PCR-based 
methods are highly sensitive but the time-consuming process for extraction and handling of RNA from eggs or second-stage juveniles (J2) may limit their application.

Developing a rapid, accurate, and efficient technique to sort (i.e., collect particles of interest for further analysis) and enumerate the developmental stages and also to assess the viability of PCN is beneficial for high-throughput analysis of nematode populations as well as bioassay tests for optimizing effective disease management decisions in commercial situations and for research. Such a method could be used frequently in assessment of chemical fumigation, biofumigation, and biocontrol agents for regulating PCN populations and keeping seed production areas below detectable levels.

The Complex Object Parametric Analyzer and Sorter (COPAS; Union Biometrica, Inc., Holliston, MA) is a large particle flow cytometer that analyzes, sorts, and collects (dispenses) objects of 20 to $1,500 \mu \mathrm{m}$ such as seed, cell clusters, fungal pellets, microencapsulated fungal spores, and often small organisms such as Caenorhabditis elegans (de Bekker et al. 2011; Delgado-Ramos et al. 2014; Pulak 2006). The COPAS allows for sorting objects in a constantly flowing stream at high speed rates of 10 to 50 objects/s. When particles or nematode specimens pass through the COPAS flow cell, several measurements are made per particle (Pulak 2006). The instrument is able to analyze and sort particles of interest based on the size (time of flight [TOF]), optical density (extinction), and fluorescent indicators. TOF is a measure of the size of an object and is determined by how long the object takes to pass the laser. Extinction is the optical density of the object passing through the flow cell. Measurements can be adjusted by changing the laser power, gain settings, or neutral density (ND) filters located before the COPAS detector. The COPAS has three bandpass filters to collect green, yellow, and red fluorescence. These filters collect fluorescence emission wavelengths in the range of 497 to 523,531 to 555 , and 602 to $628 \mathrm{~nm}$, respectively. Fluorescence is measured both as peak intensity and width, and as a complete value combined over the size of individual particle. When the instrument analyzes objects, they can be gated (i.e., regions drawn around a given set of data points with common characteristics) based on sorting parameters mentioned above, then dispensed without causing any damage to the object into Petri or multiwell plates using a gentle air-filled sorting apparatus placed after the flow cell. Because large amounts of data may be acquired by the COPAS, statistical approaches are being established for the high-throughput analysis of a large dataset.

In this study, we (i) assessed the potential of the COPAS to analyze and sort $G$. pallida cysts, eggs, and $\mathbf{J} 2$ and (ii) assessed the viability of G. pallida eggs in conjugation with fluorescent stains. The effectiveness of this instrument in separating live and dead eggs was compared with a commonly used egg viability assay using Meldola's Blue stain.

\section{Materials and Methods}

Nematode culture. Because of the quarantine status of $G$. pallida in the United States, the nematode was reared and maintained in a biosecure environment at the University of Idaho, Moscow. The population of G. pallida used in experiments was originally obtained from an infested field in Shelley, ID. Species confirmation was achieved though morphological and molecular identification (Skantar et al. 2007). In a greenhouse, the populations were reared on the susceptible potato Désirée in clay pots $(15 \mathrm{~cm}$ in diameter) filled with a sterilized sandy loam soil and sand (2:1) mix. Plants were maintained under greenhouse conditions at a range of $10^{\circ} \mathrm{C}$ night time temperature to $18^{\circ} \mathrm{C}$ day time temperature, and a photoperiod $16 \mathrm{~h}$ (day) and $8 \mathrm{~h}$ (night). After 16 weeks, cysts for experimental use were recovered by extraction from soil using the Fenwick Can method (Fenwick 1940), picked by hand under a stereomicroscope (Leica Microsystems, Wetzlar, Germany), and incubated at $4^{\circ} \mathrm{C}$ for further use.

Cysts from four greenhouse-reared sources (GP-MAQ-9-17B-RB4, GP-RBAXX-2-9-15-RB, GP-R11502-4-14-14-DS, and ORO9158-26) were used for analysis in this study. Additionally, eight samples of $G$. pallida cysts were obtained from USDA-APHIS at Idaho Falls, ID, and used to compare the viability results obtained from the COPAS with results obtained from Meldola's Blue, which is the standard protocol used by USDA-APHIS for assessment of viability (Anonymous 2014). These samples were derived from G. pallida-infested fields prefumigated (BIN00026) or postfumigated (BIN00025 numbers 1, 2, 3, 4, 5, 6, and 7) with methyl bromide or Telone as well as biofumigation cover crops.

Separation and sorting study of $\boldsymbol{G}$. pallida cyst, egg, and $\mathbf{J} 2$. Comparison of cyst separation techniques. Our initial efforts for analyzing and sorting extracted cysts from soil by the COPAS indicated large amount of debris in the samples that clogged the instrument delivery tubes or flow cell. Therefore, methods to obtain clean samples for cyst sorting and enumeration were developed. Eight methods, including ethanol and acetone flotation as well as sucrose and magnesium sulfate $\left(\mathrm{MgSO}_{4}\right.$; Fisher Scientific, Fair Lawn, NJ) combined with flotation techniques, each with three specific gravities, were used to examine their efficiency for recovering G. pallida cysts. Cysts separated by hand under a stereomicroscope were considered to be the control. In each method, $1 \mathrm{~g}$ of dried material of plant and soil debris obtained from our previous cyst extractions was added to 150 cysts of G. pallida and used for analysis.

In the acetone/ethanol flotation technique, cysts were separated from debris according to methods described by Oostenbrink (1960) and Seinhorst (1974). Briefly, the dried samples were placed in a $500-\mathrm{ml}$ volumetric flask, filled half way with $96 \%$ ethanol or acetone. The flask was thoroughly agitated and then completely filled with the ethanol or acetone. Cysts and plant debris floated to the top of the solutions and were collected using a small eyeshadow brush into a 100$\mathrm{ml}$ beaker containing tap water. Thereafter, the samples were gently filtered through Whatman filter papers and stored at room temperature $\left(22\right.$ to $\left.24^{\circ} \mathrm{C}\right)$ for $48 \mathrm{~h}$, then weighed.

In the sucrose $/ \mathrm{MgSO}_{4}$ flotation technique, cysts were isolated from debris by centrifuge according to methods described by Dunn (1969) and van Bezooijen (2006). Briefly, the samples were transferred into 50-ml conical centrifuge tubes containing $35 \mathrm{ml}$ of deionized water and incubated for $48 \mathrm{~h}$. Thereafter, $10 \mathrm{~g}$ of Kaolin (Fisher Scientific) was added to the tubes and the solutions were mixed completely, then centrifuged at $1,800 \times g$ for $4 \mathrm{~min}$. The supernatant was discarded and then replaced with three specific gravities of sucrose or $\mathrm{MgSO}_{4}$ solutions: 1.18 (sucrose or $\mathrm{MgSO}_{4}$ at $484 \mathrm{~g} /$ liter of water), 1.20 (510 g/liter of water), and 1.22 (588 g/liter of water). The pellets mixed with the solution and tubes were centrifuged at $1,800 \times g$ for 2 min. Thereafter, the supernatants (containing cysts and plant debris) obtained from different separation methods were washed over a $25-\mu \mathrm{m}$-aperture sieve, collected, stored at room temperature (22 to $24^{\circ} \mathrm{C}$ ) for $48 \mathrm{~h}$, and then weighed. The number of recovered cysts from each technique was counted under a stereomicroscope. Two trials were conducted, each with six replications.

Sorting and dispensing G. pallida cyst by using COPAS. The COPAS FP-1000 with a 15-mW, 488-nm argon-ion laser (Union Biometrica, Inc.) used in this study. For sorting G. pallida cysts, adjustments of the various components of the COPAS were made until the following optimal settings were obtained: pressure (sheath $=2.00$ psi and sample cup $=0.25$ to $0.30 \mathrm{psi}$ depending on concentration of the egg samples, diverter $=1.50 \mathrm{psi}$, and cleaning $=8.00 \mathrm{psi}$ ); acquisition (signal threshold $=500.0 \mathrm{mV}$, minimum TOF $=100 * 0.2 \mu \mathrm{S}$ ); laser power $=50.0 \mathrm{~mW}$; and extinction, green, yellow, and red gains $=1.0$. When operating the COPAS, different ND filters can be used for the appropriate samples varying in size. The 2.0-ND filter was used for analyzing and sorting cysts.

Each sample of cyst and contaminating plant debris, isolated according to the acetone or sucrose floatation technique as described in the previous section, was decanted three times over nested 850and $75-\mu \mathrm{m}$-pore sieves. The sample was subsequently transferred to the COPAS 850-ml sample cup, bringing the final volume of suspension within the cup to $500 \mathrm{ml}$ by adding deionized water. The sample was run through the COPAS and recorded data on the TOF, extinction, side scatter, and red fluorescence parameters were used to separate debris and cysts. The gated population of cysts was examined by using a profile graph that represented the shape of each object (i.e., cysts) or by visual observation of dispensed cysts under an inverted microscope at $\times 20$ (DMI 3000B; Leica Microsystems). To prevent cross-contamination, the sample recovery cup and 
delivery tubes of the instrument were rinsed first with a cleaning solution (Union Biometrica, Inc.) and then with deionized water prior to the running of each experimental sample.

Analyzing and sorting $\mathrm{G}$. pallida eggs and J2 by using COPAS. Six replicates of 10 cysts were crushed using a Tenbroeck glass tissue homogenizer, released eggs and $\mathrm{J} 2$ were disaggregated in $2 \mathrm{ml}$ of $1.0 \%$ ( $\mathrm{vol} / \mathrm{vol})$ sodium hypochlorite $(\mathrm{NaOCl})$ for $1 \mathrm{~min}$ and rinsed three times in sterile deionized water by spinning down at $4,600 \times g$ for $3 \mathrm{~min}$, and the supernatant was discarded. Eggs were subsequently transferred to a 50-ml conical centrifuge tube (sample cup) which was then filled with deionized water, bringing the total volume of the sample cup to $25 \mathrm{ml}$, and the tube was run on the COPAS equipped with the 1.0-ND filter. Adjustments of the various parameters of the COPAS were made until the following optimal settings were obtained for sorting both eggs and $\mathrm{J} 2$ : pressure (sheath $=2.00 \mathrm{psi}$ and sample cup $=0.15$ to 0.25 psi depending on concentration of the egg samples, diverter $=1.50 \mathrm{psi}$, and cleaning $=8.00 \mathrm{psi}$ ); acquisition (signal threshold $=500.0 \mathrm{mV}$, minimum $\mathrm{TOF}=60 * 0.2 \mu \mathrm{S}$ ); laser power $=50.0 \mathrm{~mW}$; and extinction, green, yellow, and red gains $=$ 1.0. In each test, approximately 1,000 to 3,000 events (i.e., eggs or $\mathrm{J} 2$ ) were detected and gated. The validity of the data from the COPAS was supported by analysis of the egg and $\mathrm{J} 2$ populations using the profile graph apparatus and visual observation under an inverted microscope. The sample recovery cup and delivery tubes were cleaned prior to the running of each experimental sample, as described above.

Dispensing efficiency of COPAS. It is critical that the dispensing process of the COPAS be accurate and highly reproducible from test to test. For proper dispensing of G. pallida eggs, J2, and cysts, adjustments of two parameters on the COPAS were made until the following optimal settings were selected: drop width $=5.00 \mathrm{mS}$, sort delay = $12.00 \mathrm{mS}$ for both eggs and $\mathrm{J} 2$, drop width $=12.00 \mathrm{mS}$, and sort delay $=$ $14.00 \mathrm{mS}$ for cysts. The dispensing efficiency (purity) was examined by dispensing six replicates of 1,000 eggs, $\mathbf{J} 2$, or cysts from defined gates into multiwell plates. Numbers of dispensed nematode individuals were counted and the purity was calculated as (number of nematodes detected/ total number of nematodes detected) $\times 100$.

Viability study of $\boldsymbol{G}$. pallida egg. Viability of G. pallida $e g g$ stained with Meldola's Blue. The viability of three cyst nematode populations (GP-RBAXX-2-9-15-RB, GP-R11502-4-14-14-DS, and ORO9158-26) that were used in subsequent COPAS tests was examined with a Meldola's staining assay. This assay was used as a reference to compare the results of egg viability obtained from the COPAS (see below). Four replicates of 10 cysts from each population were soaked in water for $24 \mathrm{~h}$, crushed, and surface disinfested in $1.0 \% \mathrm{NaOCl}$ for $1 \mathrm{~min}$ before being treated with Meldola's Blue
A
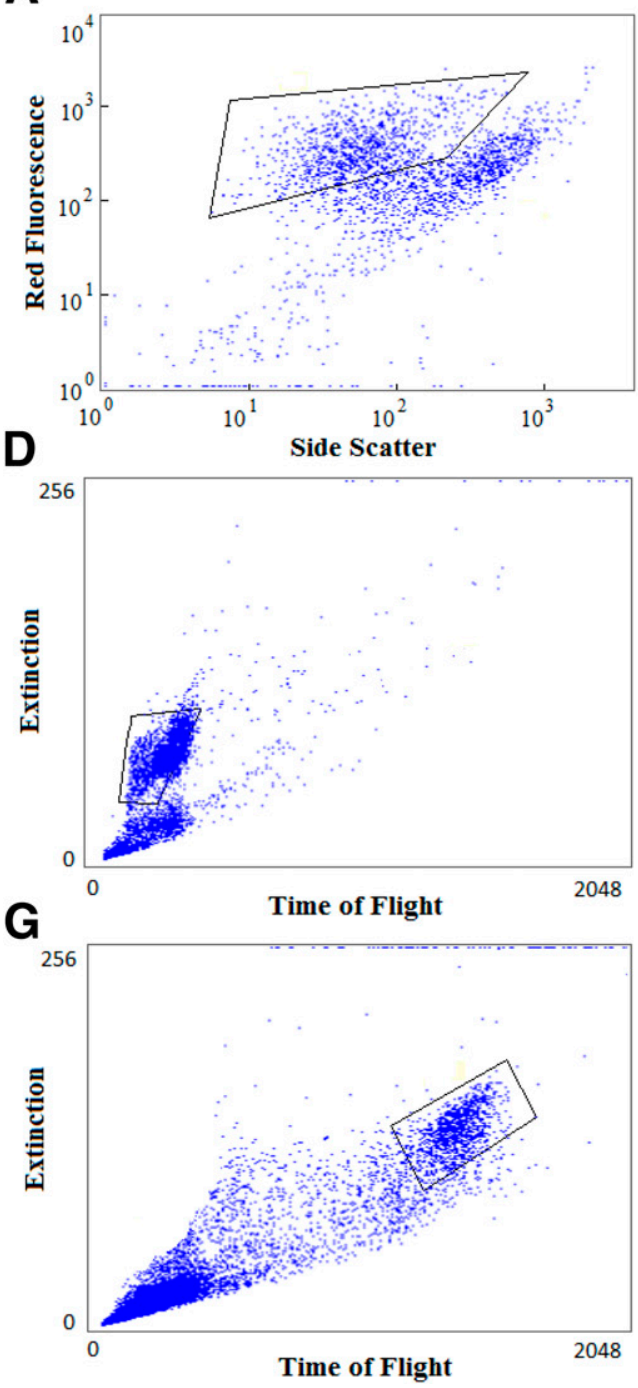

B

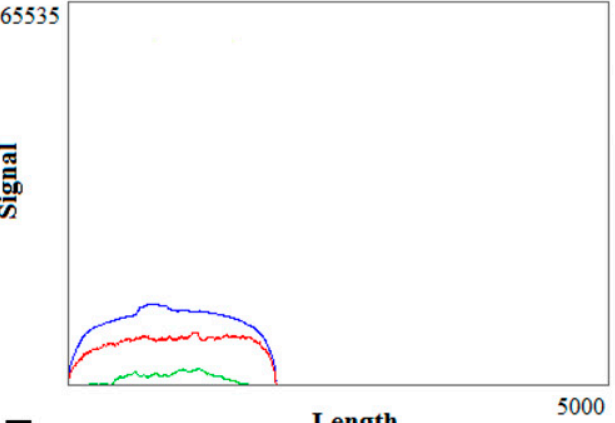

E
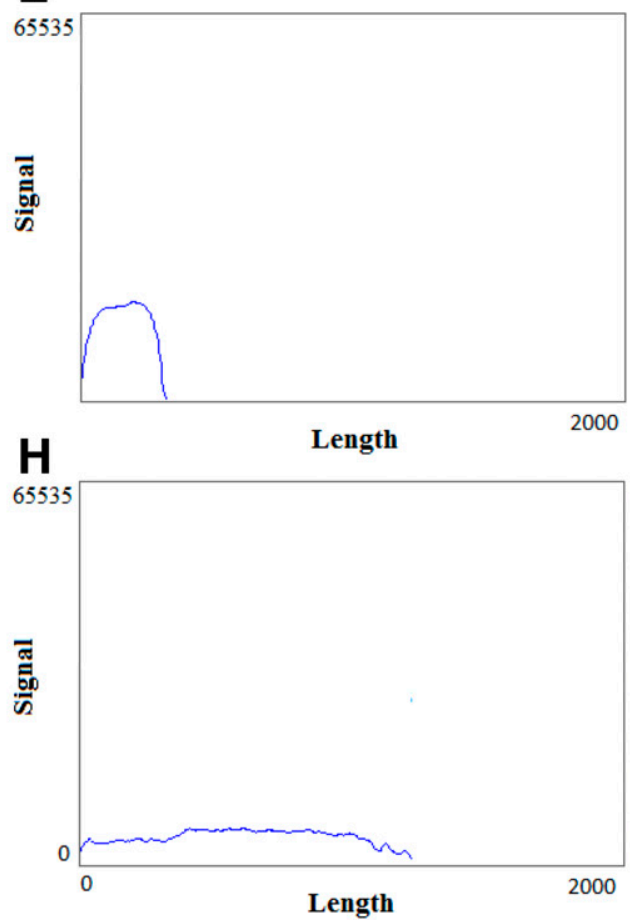

C

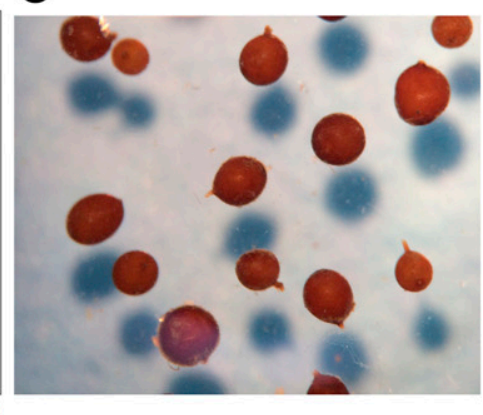

$\mathbf{F}$

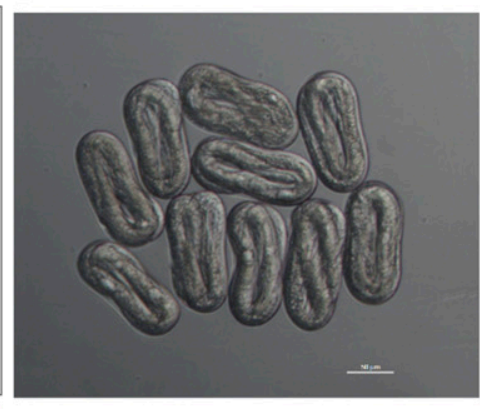

I

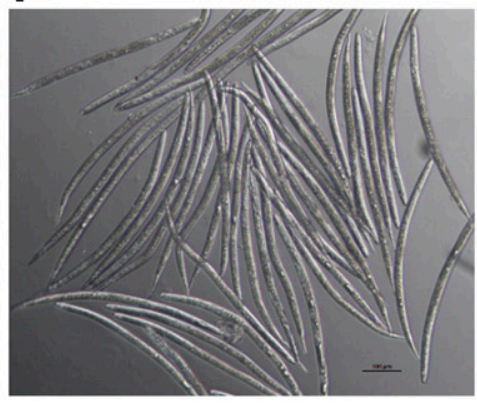

Fig. 1. Globodera pallida A to C, eggs; D to F, second-stage juveniles; and G to I, cysts analyzed by the COPAS large particle flow cytometer. Red fluorescence (615 nm) was excited using the 488-nm excitation laser and was based on the fluorescence of a passing object. Dot plots represent sort gates selected using different sorting criteria; profile graphs display the profiling measurements and shapes of single egg, second-stage juvenile, or cyst; and microscopic images represent dispensed nematode individuals into wells of a 96-well plate and photographed. 
(Spectrum Chemical, New Brunswick, NJ). Eggs were rinsed three times in sterile deionized water by spinning down at $460 \times g$ for $3 \mathrm{~min}$ and discarding the supernatant. Meldola's Blue (500 $\mu \mathrm{l}$ of $0.05 \%$ [wt/vol]) was added to $500 \mu \mathrm{l}$ of sterile deionized water in a 2-ml Eppendorf tube containing nematode eggs. Sample tubes then were shaken gently by inverting the tubes for $5 \mathrm{~min}$ and incubated at $25^{\circ} \mathrm{C}$ in the dark for 7 days. After incubation, eggs were transferred onto a $25-\mu \mathrm{m}$-aperture sieve and rinsed with deionized water to remove stain residue. Assessments of egg viability was conducted visually by using an inverted microscope. For this purpose, 100 stained eggs were dispensed by the COPAS into a 96-well plate (1 egg per well, except the last four wells that contained 2 eggs) from a sort gate of an egg population; then, the live and dead eggs were counted.
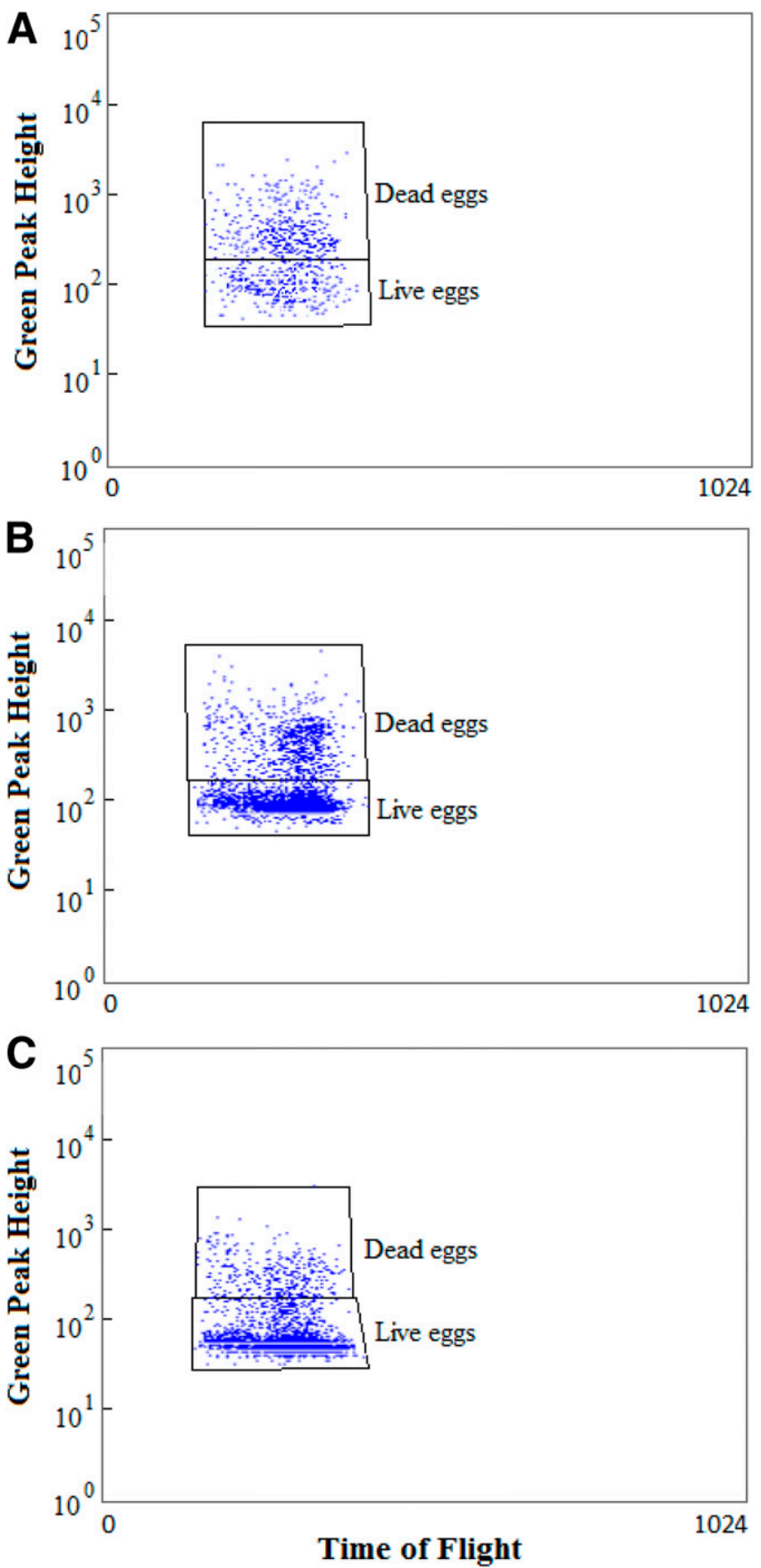

Fig. 2. Examples of assessment of viability of Globodera pallida in bioassay I. Eggs from three populations of A, GP-RBAXX-2-9-15-RB; B, GP-R11502-4-14-14-DS; and C, ORO9158-26 were stained with SYTOX Green and analyzed by the COPAS large particle flow cytometer. Two sort gates, corresponding to live (lower gate) and dead (upper gate) eggs, can be readily separated.
Those eggs that did not stain were considered alive; partially and wholly stained eggs were considered dead. The percentage of eggs estimated to be live was calculated as (number of unstained eggs/ total number of stained and unstained eggs) $\times 100$. The experiment was repeated once and treatments (stained eggs) in each replicate were randomized prior to analysis by the COPAS.

Viability of G. pallida egg stained with fluorescence stains. To optimize which sorting approach (by combination of the COPAS and SYTOX Green stain) yielded the most accurate viability assessment and separated live from dead eggs, a preliminary experiment was conducted. In all, 10 cysts of a population (GP-MAQ-9-17-B-RB4) of $G$. pallida were soaked in water for $24 \mathrm{~h}$, transferred to a 2-ml Eppendorf tube with $500 \mu \mathrm{l}$ of deionized water, then crushed gently using a Tenbroeck glass tissue homogenizer. Eggs were surface disinfested in $1 \mathrm{ml}$ of $1.0 \%$ (vol/ $/ \mathrm{vol}) \mathrm{NaOCl}$ for $1 \mathrm{~min}$ and rinsed three times in sterile deionized water by spinning down at 4,600 $\times g$ for 3 min and discarding the supernatant. After the final wash, the supernatant was removed and the nematode eggs were stained with two fluorescent stains, SYTOX Green nucleic acid (Thermo Fisher Scientific, Eugene, OR) and Live/Dead fixable green dead cell stain kits (Thermo Fisher Scientific). Four working solutions of SYTOX Green (concentration of 5, 10,20 , and $50 \mu \mathrm{M}$ ) and one concentration of Live/Dead stain kit ( $25 \mu \mathrm{g}$ per $50 \mu \mathrm{l}$ of dimethyl sulfoxide) were prepared as per the manufacturer's instructions and used to stain the nematode eggs for different time periods $(1,2,4,8,12,24$, and $36 \mathrm{~h})$ in the dark at room temperature $\left(22\right.$ to $\left.24^{\circ} \mathrm{C}\right)$. Our preliminary examination to separate live and dead eggs stained with the Live/Dead stain kit by using the COPAS was not successful. In fact, fluorescence from stained eggs with the Live/Dead stain kit was too weak to be detected by the COPAS. In contrast, eggs treated with SYTOX Green emitted green fluorescence of $523 \mathrm{~nm}$ that was detected in the green channel of the COPAS. Additionally, our preliminary results showed that the egg viability steadily increased as the incubation times increased with no significant differences observed between incubation times of 12,24 , and $36 \mathrm{~h}$. Of these two fluorescence stains, SYTOX Green $(50 \mu \mathrm{M}$ for $24 \mathrm{~h}$ of incubation) was selected for use in further experiments. Several adjustments were performed to optimize the COPAS function in assessing egg viability by changing the sort gates for live and dead eggs, followed by dispensing eggs from both defined gates in 96 -well plates and examining at $\times 40$ using an inverted microscope equipped with a fluorescence Y5 filter.

After obtaining optimum sorting parameters as well as fluorescence levels at which live and dead eggs could be differentiated, the viability of G. pallida was assessed in two separate bioassays. In the first bioassay (bioassay I), three sources of greenhouse-reared cysts (GPRBAXX-2-9-15-RB, GP-R11502-4-14-14-DS, and ORO9158-26) were examined. The reliability of measuring nematode viability in bioassay I was confirmed in bioassay II by assessment of eight cyst populations (BIN00026-prefumigation and BIN00025-postfumigation numbers $1,2,3,4,5,6$, and 7), obtained from USDA-APHIS, Idaho Falls, ID. In both bioassays, four replications of 10 cysts from each cyst population were crushed gently in 2-ml Eppendorf tubes. Intact eggs were separated from empty eggs, eggshells, and contaminating debris by suspending each sample in $2 \mathrm{ml}$ of $35 \%$ sucrose solution. Eggdebris mixture $(1 \mathrm{ml})$ was added to $1 \mathrm{ml}$ of $70 \%$ sucrose in a 2-ml tube. The tube was centrifuged at $1,260 \times g$ for $5 \mathrm{~min}$ and, thereafter, the eggs were collected from the top $0.5 \mathrm{ml}$ using a pipette and transferred to new 2-ml tubes containing $1 \mathrm{ml}$ of $1.0 \% \mathrm{NaOCl}$. After $1 \mathrm{~min}$, the eggs were washed in sterile water, suspended in a solution of SYTOX Green $(50 \mu \mathrm{M})$, incubated for $24 \mathrm{~h}$ in the dark at room temperature (22 to $24^{\circ} \mathrm{C}$ ), and subsequently transferred to the COPAS 50-ml sample cup. The sample cup was then filled with deionized water, bringing the total volume of the cup to $30 \mathrm{ml}$. Samples were analyzed and number of live and dead eggs was recorded. Prior to the running of each experimental sample, delivery tubes and sample recovery cup were washed as described in the previous section. Two trials were conducted in each bioassay described above and replicates for each of the cyst population were randomized prior to analysis. The data on percentage of live eggs obtained from bioassay I and II were compared with those from Meldola's Blue staining assays conducted in the present study and by USDA-APHIS, respectively. 
Statistical analysis. Data were plotted on the COPAS software using two-dimensional dot plots to recognize appropriate sorting parameters that characterized individual eggs, J2, and cysts. During data acquisition, the information on total number of objects detected, percentage of the gate that contains total objects, percentage of the parent gate, as well as mean and coefficient of variation of gated objects were observed. Prior to data analysis, the assumptions for normality of residuals were examined and corrected when necessary based on the Shapiro-Wilk test. Homogeneity of variance was tested using Bartlett's test. Data for the two trials for each cyst separation study $(n=12)$ and egg viability study $(n=8)$ were combined because there was no difference $(P>F=1.0)$ between means of repeat trials. In the cyst separation study, data on the percentage of recovered cyst and dry weight of debris were analyzed using a one-way analysis of variance, where extraction method was considered as a fixed effect and replication and repeat trial as random effects. When treatment means were significant at $P<0.05$, Fisher's least significant difference test was used. To examine whether percentage of viability of eggs stained with Meldola's Blue differed from those stained with SYTOX Green in the viability study, a two-sample $t$ test assuming equal variances was performed. All statistical analyses were performed using the software SAS 9.3 (SAS Institute Inc., Cary, NC).

\section{Results}

Separation and sorting study of G. pallida cysts, eggs, and J2. Analyzing and sorting G. pallida cysts. The recovery percentage of the $G$. pallida cysts from plant and soil debris was greater in ethanol $(94 \%)$, acetone (93\%), and centrifuge + sucrose (sp. gr. 1.18) $(92 \%)$ compared with other cyst separation techniques used. However, there were no significant differences in cyst recovery among ethanol, acetone, and centrifuge + sucrose (sp. gr. 1.18 ) techniques (Table 1). The amount of debris associated with cysts in centrifuge $+\mathrm{MgSO}_{4}$ (sp. gr. 1.18 and 1.22) was significantly less than those associated with other techniques used (Table 1). G. pallida cysts were sorted and distinguished from plant debris on the COPAS based on side scatter (laser reflection) and red fluorescence $(615 \mathrm{~nm})$ parameters (Fig. 1A to C). Side scatter measures light scatter and is proportional to the internal complexity of the particle being sorted, and red fluorescence parameter was based on the fluorescence of a passing object. By the COPAS, large cysts with a maximum length and width of 838 and $626 \mu \mathrm{m}$, respectively, were sorted and analyzed. The purity of the sorted cysts by the COPAS was approximately $94 \%$.

Analyzing and sorting G. pallida eggs and J2. A sort gate was set on the COPAS to detect and distinguish either G. pallida eggs (Fig. 1D to F) or J2 (Fig. 1G to I) from debris based on extinction (optical density) and TOF (size) parameters. We also were able to separate eggs from $\mathrm{J} 2$ using the same sorting criteria with no fluorescent dye required. The purity of the sorted eggs and $\mathrm{J} 2$ was approximately 96 and $95 \%$, respectively. The COPAS was unable to differentiate between live and dead J2 stained with SYTOX Green based on all sorting criteria.

Viability study of $\boldsymbol{G}$. pallida eggs. Analyzing egg viability by using Meldola's Blue stain. Meldola's Blue is a nonfluorescent dye that
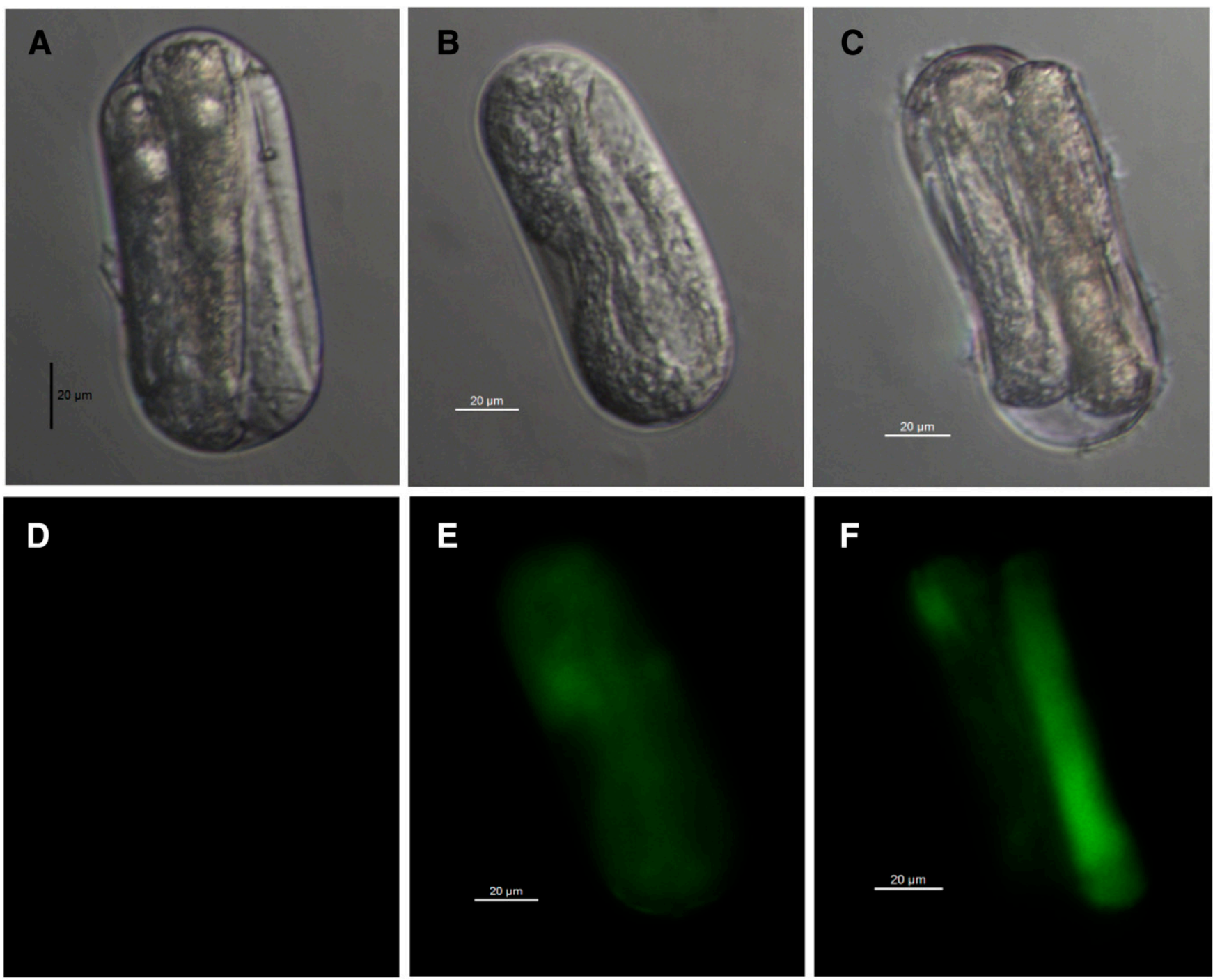

Fig. 3. Eggs of Globodera pallida stained with SYTOX Green, sorted and dispensed by the COPAS large particle flow cytometer. Brightfield images of A, live and B and C, dead eggs; and fluorescence images of $\mathbf{D}$, live and $\mathbf{E}$ and $\mathrm{F}$, dead eggs. 
readily enters cell membranes of dead eggs. In an effort to separate live from dead eggs of $G$. pallida stained with Meldola's Blue on the COPAS, data were analyzed based on red fluorescence and TOF sorting parameters. Two gates with no or dim red fluorescence and high red fluorescence, representing live and dead eggs, respectively, were set (data not shown). However, the accuracy of sorted eggs from both live and dead gates was $<80 \%$, indicating the inaccuracy in measuring the viability of eggs stained with Meldola's Blue.

Analyzing egg viability by using SYTOX Green stain. SYTOX Green is not able to cross compromised membranes of live eggs; therefore, it stains dead eggs upon binding nucleic acids. Dead eggs exhibit green fluorescence with an excitation and emission peak of 504 and $523 \mathrm{~nm}$, respectively, when excited by a 488-nm argon-ion laser. Based on green fluorescence and TOF parameters, we were able to detect both stained and unstained eggs. The acquired data were then separated based on intensity of fluorescence using green peak height and TOF parameters. Eggs having green fluorescence peaks $<190$ were considered to be alive and those with green fluorescence peaks $>190$ to be dead (Fig. 2). These results were confirmed when heat-killed eggs of G. pallida stained with SYTOX Green had fluorescence intensity $>190$, suggesting a clear differentiation between dead and live eggs that had weak or no florescence (data not shown). Additionally, the results were confirmed by dispensing eggs from both gates and observing them under a fluorescence microscope. Fluorescing dead eggs were bright green, whereas live eggs exhibited no fluorescence (Fig. 3).

In bioassay I, the percentage of live eggs of G. pallida stained with SYTOX Green was greater in only one cyst population (GP-R115024-14-14-DS) than those stained with Meldola's Blue (Fig. 4). The percentage of live eggs, however, was greater than those stained with Meldola's Blue $(P<0.05)$ when data across all three populations of cyst were pooled over the two trials. In bioassay II, the percentage of live eggs stained with SYTOX Green in all prefumigant and postfumigant cyst populations was significantly greater $(P<0.001)$ than those stained with Meldola's Blue (Table 2). We observed that both live and empty eggs were often seen as dark objects by the fluorescence microscope and, therefore, were difficult to distinguish from each other. Similar results were observed when the samples were analyzed by the COPAS.

\section{Discussion}

Analyzing and sorting $G$. pallida development stages. To our knowledge, this is the first detailed investigation using the COPAS large particle flow cytometer to analyze cysts of plant-parasitic nematodes. The results presented in this study showed that the COPAS can successfully isolate, sort, and dispense pure populations of $G$. pallida cysts. Fluorescence-activated cell sorting is a highly specialized technology developed for the isolation of subpopulations of particles in a heterogeneous population based on fluorescence intensity (Pulak 2006). The most widely used application of standard flow cytometers has been to analyze and sort small particles (5 to $200 \mu \mathrm{m}$ in size) such as bacteria (Lebaron et al. 1998; Roth et al. 1997) or nematode (e.g., C. elegans) eggs (Schmidt et al. 2016). We developed a simple but effective protocol to isolate of G. pallida cysts from other large particles (plant or root debris) contained in samples extracted from the soil. The method was primarily based on the properties of cysts, which allows them to float in acetone or sucrose solutions. The separated cysts and debris were then purified more using a sieving method to produce samples with the lowest amount of debris to enable successful sorting of cysts of variable sizes on the COPAS. Because the nematode cysts and plant debris differ in size and shape, they were separated and sorted based on the different light-scattering properties that they exhibited. By using the COPAS, samples with 1,000 cysts could be sorted to $94 \%$ purity and analyzed in 20 to $25 \mathrm{~min}$. We analyzed the cyst samples at low rates ( 1 to 5 events/s) of the sample flow by adjusting the sample cup pressure on the COPAS, allowing appropriate number of objects to pass through the flow cell. The drawbacks of the existing PCN processing techniques for detection and population assessment of PCN affect the overall efficiency because this task is extremely labor intensive and can require several full-time technicians. The processing time and manual labor required for population assessment of PCN will be significantly decreased by using the COPAS. However, analyzing nematode cysts on the COPAS can be occasionally problematic if cyst samples contain high amounts of organic matter.

There are a few examples of application of flow cytometers to isolate and sort nematode eggs (Schmidt et al. 2016; Tylka et al. 1993), embryos (Stoeckius et al. 2009), and larvae (Fernandez et al. 2012). The ability to sort the eggs of plant-parasitic nematodes using flow cytometry was first described by Tylka et al. (1993). In their study, stage-specific analysis and sorting of $H$. glycines eggs was conducted using a Coulter EPICS 752 cell sorter based on object size (forward angle) and granularity (side scatter) parameters. Fernandez et al. (2012) developed a protocol for automatic selection of viable J1 of C. elegans using a standard fluorescence-activated cell sorter equipped with a $100-\mu \mathrm{m}$ flow cell. In the present study, the COPAS was successfully used for sorting, enumerating, and dispensing pure populations of both individual eggs and $\mathrm{J} 2$ of G. pallida. Of particular capability of the COPAS for nematode sorting was the initial separation of the eggs and $\mathbf{J} 2$ from large unwanted particulates such as plant debris. The COPAS sorted and enumerated 5,000 or more eggs or J2 from a mixed population with $96 \%$ purity in 5 to $10 \mathrm{~min}$. This rate of collection could make this technique ideal for preparing known population densities of nematodes to conduct various applied and fundamental nematology research. Flow cytometry has played a crucial role in unraveling the obscurities of some problems in applied biology and nematology. Clover (2009) stated that sorting C. elegans larvae by the COPAS had no impact on the survival rate of the organism, suggesting that this instrument can be easily used for analyzing and sorting PCN developmental stages with no negative impact on the nematode infectivity.

Assessing the viability of $G$. pallida. Assessment of the pathogenicity of plant-pathogenic nematodes in host plants regularly requires that live and dead nematodes be separated from each other. When nematodes eggs are examined rather than motile juvenile and adult individuals, it is challenging to determine whether a nematode is viable or nonviable. A number of procedures have been used by researchers to determine which egg individuals are live and which are not using vital stains. However, variable outcomes have been reported for some of these stains (Kroese et al. 2011; Ogiga and Estey 1974; Shepherd 1962). In the present study, the results showed that the COPAS can be used as an in vitro test to determine the viability of G. pallida. In an effort to improve cost effectiveness and simplicity, we used different populations of the cysts to examine the relevance of two fluorescence dyes (Live/Dead stain kit and SYTOX Green) in discriminating live eggs from dead eggs. The Live/Dead stain kit is a cell-permeable red or green fluorescent stain that reacts

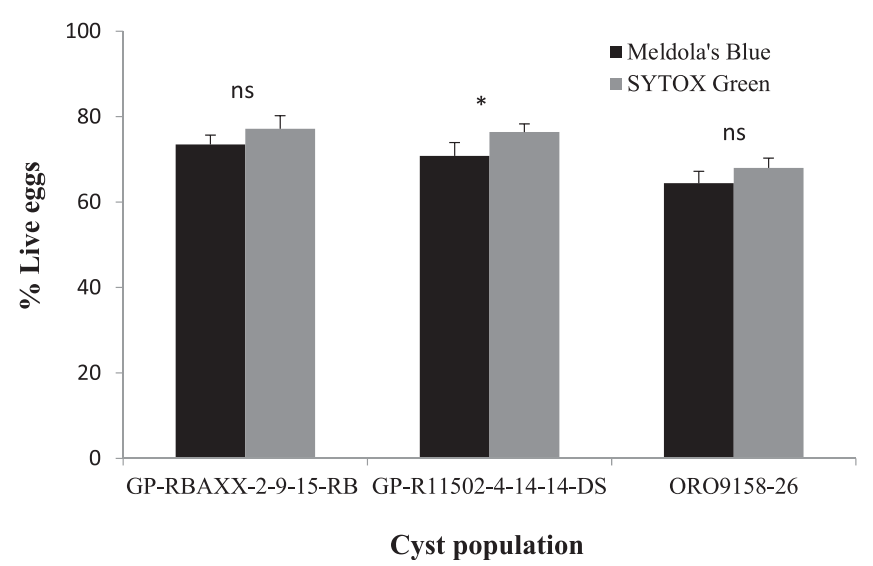

Fig. 4. Assessment of viability of Globodera pallida eggs stained with Meldola's Blue and SYTOX Green. Eggs stained with Meldola's Blue were assessed visually by using an inverted microscope, whereas those stained with SYTOX Green by the COPAS large particle flow cytometer. Comparison of means between staining assays was performed using $t$ tests; ns $=$ no significant difference and * indicates $P<0.05$. Each column signifies the means \pm standard error $(n=8)$. 
with amines both in the interior and on the cell surface. Cells treated by this reactive stain will exhibit green and red fluorescence with emission peaks of about 530 and $630 \mathrm{~nm}$, respectively, upon excitation by a 488-nm laser. In our study, fluorescence from stained $G$. pallida eggs with the Live/Dead stain kit was too weak to be detected by the COPAS. In contrast, SYTOX Green penetrated eggs with permeabilized shells and bound nucleic acids, staining dead eggs more brightly and stably than the Live/Dead stain kit. In our study, egg viability was examined $24 \mathrm{~h}$ after staining with SYTOX Green, which is when the maximum level of fluorescence was reached in a 24-h period. Intensity of green fluorescence and percentage of live eggs, however, did not significantly increase despite the prolonged incubation of stained eggs for an additional $12 \mathrm{~h}$. We examined several different sorting parameters such as light scattering and fluorescence measurements to determine which sorting approach gave the best separation of live and dead eggs. We found that green peak height and TOF were optimal for assessing the viability of G. pallida.

In the present study, the numbers of live eggs stained with SYTOX Green was $6.1 \%$ greater than those stained with Meldola's Blue. The reliability of bioassay I was confirmed in bioassay II by assessment of one pre- and seven postfumigated cyst populations of G. pallida. With cyst samples from USDA-APHIS, the difference in the percentage of viable eggs examined with SYTOX Green and Meldola's Blue was notable. SYTOX Green staining led to the detection of as much as 7 to $14.6 \%$ more live eggs than did Meldola's Blue. We found that SYTOX Green overestimated the percentage of live eggs when the population contained a high number of empty eggs because empty eggs did not take up the stain and were detected and counted by the COPAS as living eggs. Our visual observations under a fluorescence microscope confirmed that intact eggshells or empty eggs were not permeable to SYTOX Green. We assume that chemical treatment of PCN-infested soils could cause degradation of nucleic acids of eggs with no impact on the integrity of shells. Therefore, staining empty eggs with SYTOX Green resulted in weak fluorescence that corresponded to live eggs. In other words, the COPAS detected dead eggs with a low fluorescence and counted them as live eggs because they appeared in the defined gate corresponding to that of live eggs. Due to this fact, the percent viability of $G$. pallida eggs increased in the samples from USDA-APHIS that had greater numbers of empty eggs. Our results are in agreement with Lebaron et al. (1998), who reported that staining bacteria with SYTOX Green resulted in underestimation of dead cells in populations containing cells with damaged nucleic acids or membranes.

Developing a reliable and simple technique to separate live from dead eggs is vital for high-throughput screening of chemical nematicides or biological compounds against PCN. Currently, plant infectivity bioassays seem most reliable to determine viability of potato cyst nematodes but such assays are time consuming and labor intensive. Back et al. (2004) reported that staining G. rostochiensis and G. pallida eggs with Meldola's Blue resulted in a higher estimation of viability for fumigated and nonfumigated populations of both nematodes when compared with hatching assays. Fluorescent-based methods in combination with flow cytometry have been widely used for assessment of viability of microorganisms from different environmental samples (Roth et al. 1997; Schmidt et al. 2016). Results presented herein established that application of the COPAS in combination with nucleic-acid-binding dyes can provide a quantitative, precise, and sensitive technique to assess live and dead eggs of G. pallida. However, our results suggest that the use of SYTOX Green for assessment of egg viability of PCN should be done with caution because of the inability to stain empty eggs. We assume that the accuracy of this technique could be enhanced by using fluorescence dyes with the ability to stain live eggs rather than dead eggs. More recently, Schmidt et al. (2016) developed a procedure for flow cytometric detection of viable eggs of Nippostrongylus brasiliensis and stated that SYTO17 nucleic acid stain had the ability to stain the live nematode eggs.

In conclusion, we have developed an effective recovery method based on the combination of standard cyst extraction techniques and the COPAS for sorting, counting, and dispensing different developmental stages of $G$. pallida. Our simple and quick method can replace the current time-consuming, manual labor procedures of analyzing, sorting, and enumerating PCN under a microscope. We also have established an efficient technique based on the COPAS and egg staining with a florescence dye, SYTOX Green, for testing the viability of G. pallida eggs. Although this method has some limitations regarding the estimation of viable eggs, it clearly has potential to assess the viability of PCN. The performance of egg viability

Table 2. Percent viability of eight populations of Globodera pallida after staining with SYTOX Green and analyzing by COPAS large particle flow cytometer $^{\mathrm{w}}$

\begin{tabular}{lccc}
\hline & \multicolumn{2}{c}{ Live eggs $(\%)^{\mathbf{x}}$} & \\
\cline { 2 - 3 } Cyst population & & \\
\cline { 2 - 3 } & Meldola's Blue & SYTOX Green $^{\mathbf{t}}$ & $\boldsymbol{t}$ Test $^{\mathbf{z}}$ \\
\hline BIN26 & 17.7 & 30.1 & $* *$ \\
BIN25-1 & 5.8 & 19.3 & $* * *$ \\
BIN25-2 & 1.2 & 15.8 & $* * *$ \\
BIN25-3 & 6.6 & 18.2 & $* * *$ \\
BIN25-4 & 0.5 & 14.1 & $* * *$ \\
BIN25-5 & 0.6 & 15.0 & $* * *$ \\
BIN25-6 & 0.0 & 14.6 & $* * *$ \\
BIN25-7 & 0.4 & 13.7 & $* * *$ \\
\hline
\end{tabular}

${ }^{\mathrm{w}}$ Data were compared with known rates of egg viability obtained using a Meldola's Blue staining assay.

${ }^{x}$ Eggs stained with Meldola's Blue were assessed visually by using a microscope whereas those stained with SYTOX Green by the COPAS large particle flow cytometer. Each value signifies the means of $n=8$ (SYTOX Green) and $n=6$ (Meldola's Blue).

y Cyst populations obtained from the United States Department of AgricultureAnimal and Plant Health Inspection Service. BIN26 = prefumigated cysts and BIN25 (numbers 1 to 7 ) = postfumigated cysts with methyl bromide or Telone as well as biofumigation cover crops.

${ }^{\mathrm{z}}$ Comparison of viability variables between staining assays using $t$ tests; $* *$ and *** indicate $P<0.01$ and 0.001 , respectively.

Table 1. Recovery of Globodera pallida cysts from plant and soil debris using various separation techniques ${ }^{y}$

\begin{tabular}{lcc}
\hline Separation technique & & $\begin{array}{c}\text { Dry weight of debris } \\
\text { associated with cyst (mg) }\end{array}$ \\
\hline Control (hand picking) & Cyst recovery (\%) & $1,000 \pm 12.3 \mathrm{a}$ \\
Ethanol & $95 \pm 0.9 \mathrm{a}$ & $21.2 \pm 2.8 \mathrm{~b}$ \\
Acetone & $94 \pm 0.7 \mathrm{a}$ & $15.0 \pm 1.4 \mathrm{c}$ \\
Centrifuge + sucrose (sp. gr. 1.18) & $93 \pm 0.6 \mathrm{a}$ & $15.2 \pm 1.9 \mathrm{c}$ \\
Centrifuge + sucrose (sp. gr. 1.20) & $92 \pm 2.9 \mathrm{a}$ & $19.4 \pm 1.6 \mathrm{~b}$ \\
Centrifuge + sucrose (sp. gr. 1.22) & $85 \pm 2.0 \mathrm{~b}$ & $22.1 \pm 2.9 \mathrm{~b}$ \\
Centrifuge $+\mathrm{MgSO}_{4}$ (sp. gr. 1.18) & $91 \pm 2.0 \mathrm{ab}$ & $5.4 \pm 2.3 \mathrm{e}$ \\
Centrifuge $+\mathrm{MgSO}_{4}$ (sp. gr. 1.20) & $64 \pm 0.7 \mathrm{c}$ & $9.2 \pm 3.4 \mathrm{~d}$ \\
Centrifuge $+\mathrm{MgSO}_{4}$ (sp. gr. 1.22) & $66 \pm 1.4 \mathrm{c}$ & $6.6 \pm 2.4 \mathrm{e}$ \\
\hline
\end{tabular}

${ }^{y}$ Each value indicates the mean \pm standard error of six replicates. Values in each column followed by the same letter are not significantly different according to Fisher's protected least significant difference test $(P=0.05)$.

${ }^{\mathrm{z}} \mathrm{Sp}$. gr. = specific gravity of sucrose or $\mathrm{MgSO}_{4}$ solutions. 
and quantification assays for PCN is increased compared with oldfashioned egg staining and hatching assays. Development of a precise quantitative viability assay would be useful for all circumstances where information on the pathogenicity or infectivity of PCN is desired. This method would also be of particular advantage to growers that treat PCN-infested fields with fumigant nematicides. Further investigations of other fluorescence dyes with the capability of staining live eggs and not dead eggs remain necessary for precise quantification of PCN viability.

\section{Acknowledgments}

We thank T. Gresham, USDA-APHIS, for providing G. pallida cyst samples; and members of PCN Laboratory A. Gray, E. Forsberg, A. McGinnis, and R. Kooliyottil at the University of Idaho for their technical assistance in this study.

\section{Literature Cited}

Anonymous. 2014. Canada and United States: Guidelines on surveillance and phytosanitary actions for the potato cyst nematodes Globodera rostochiensis and Globodera pallida. Online publication. https://www.aphis.usda.gov/ plant_health/plant_pest_info/nematode/downloads/potato_guidelines.pdf

Back, M. A., Heath, W., Edwards, S., Haydock, P. P. J., Grove, I. G. G., and Minnis, S. 2004. Development of a robust assay to assess the viability of potato cyst nematodes. Department for Environment, Food and Rural Affairs. CSG 15.

Beniers, J. E., Been, T. H., Mendes, O., van Gent-Pelzer, M. P. E., and van der Lee, T. A. J. 2014. Quantification of viable eggs of the potato cyst nematodes (Globodera spp.) using either trehalose or RNA-specific real-time PCR. Nematology 16:1219-1232.

Christoforou, M., Pantelides, I. S., Kanetis, L., Ioannou, N., and Tsaltas, D. 2014. Rapid detection and quantification of viable potato cyst nematodes using qPCR in combination with propidium monoazide. Plant Pathol. 63:1185-1192.

Clover, R. 2009. Effect of SYTOX staining in combination with manual sorting or COPAS technology sorting on the mean survival of wild-type $C$. elegans. COPAS Tech. Note TN01. Online publication. http://www.unionbio.com/ documents/tn01.pdf

de Bekker, C., van Veluw, G. J., Vinck, A., Wiebenga, L. A., and Wosten, H. A. 2011. Heterogeneity of Aspergillus niger microcolonies in liquid shaken cultures. Appl. Environ. Microbiol. 77:1263-1267.

Delgado-Ramos, L., Marcos, T., Ramos-Guelfo, M., Sanchez-Barrionuevo, L., Smet, F., Chavez, S., and Canovas, D. 2014. Flow cytometry of microencapsulated colonies for genetics analysis of filamentous fungi. Genes Genom. Genet. 4 (G3):2271-2278.

Devine, K. J., Byrne, J., Maher, N., and Jones, P. W. 1996. Resolution of natural hatching factors for golden potato cyst nematode, Globodera rostochiensis. Ann. Appl. Biol. 129:323-334.

Dunn, R. A. 1969. Extraction of Heterodera species from soils by centrifugation in high density solutions. J. Nematol. 1:7.

Ebrahimi, N., Viaene, N., and Moens, M. 2015. Optimizing trehalose-based quantification of live eggs in potato cyst nematodes (Globodera rostochiensis and G. pallida). Plant Dis. 99:947-953.

Evans, K., and Stone, A. R. 1977. A review of the distribution and biology of the potato cyst-nematodes Globodera rostochiensis and G. pallida. PANS/Cent. Overseas Pest Res. 23:178-189.

Fenwick, D. W. 1940. Methods for the recovery and counting of cysts of Heterodera schachtii from soil. J. Helminthol. 18:155-172.
Fernandez, A. G., Bargmann, B. O. R., Mis, E. K., Edgley, M. L., Birnbau, K. D. and Piano, F. 2012. High-throughput fluorescence-based isolation of live C. elegans larvae. Nat. Protoc. 7:1502-1510.

Kaemmerer, D. 2012. Detection of viable potato cyst nematodes (Globodera rostochiensis and $G$. pallida) by reverse transcription PCR. J. Plant Dis. Prot. 119:100-106.

Kroese, D., Zasada, I. A., and Ingham, R. E. 2011. Comparison of Meldola's Blue staining and hatching assay with potato root diffusate for assessment of Globodera sp. egg viability. J. Nematol. 43:182-186.

Lebaron, P., Catala, P., and Parthuisot, N. 1998. Effectiveness of SYTOX Green stain for bacterial viability assessment. Appl. Environ. Microbiol. 64:26972700.

Meyer, S. L. F., Sayre, R. M., and Huettl, R. N. 1988. Comparison of selected stains for distinguishing between live and dead eggs of the plant-parasitic nematode Heterodera glycines. Proc. Helminthol. Soc. Wash. 55:132-139.

OEPP/EPPO. 2013. Globodera rostochiensis and Globodera pallida. Bull. OEPP/EPPO Bull. 43:119-138.

Ogiga, I. R., and Estey, R. H. 1974. The use of Meldola Blue and Nile Blue A, for distinguishing dead from living nematodes. Nematologica 20:271-276.

Oostenbrink, M. 1960. Estimating nematode populations by some selected methods. Pages 85-102 in: Nematology. J. N. Sasser and W. R. Jenkins, eds. The University of North Carolina Press, Chapel Hill.

Porter, J., Edwards, C., and Pickup, R. W. 1995. Rapid assessment of physiological status in Escherichia coli using fluorescent probes. J. Appl. Bacteriol. 79: 399-408.

Pulak, R. 2006. Techniques for analysis, sorting, and dispensing of C. elegans on the COPAS flow-sorting system. Methods Mol. Biol. 351:275-286.

Roth, B. L., Poot, M., Yue, S. T., and Millard, P. J. 1997. Bacterial viability and antibiotic susceptibility testing with SYTOX Green nucleic acid stain. Appl Environ. Microbiol. 63:2421-2431.

Schmidt, A., Bouchery, T., Le Gros, G., and Price, K., 2016. Large particle sorting to isolate live parasitic nematode eggs. Curr. Protoc. Cytom. 76:11.21.1-11.21 15

Seinhorst, J. W. 1974. Separation of Heterodera cysts from dry organic debris using ethanol. Nematologica 20:367-369.

Shepherd, A. M. 1962. New blue R, a stain that differentiates between living and dead nematodes. Nematologica 8:201-208.

Skantar, A. M., Handoo, Z. A., Carta, L. K., and Chitwood, D. J. 2007. Morphological and molecular identification of Globodera pallida associated with potato in Idaho. J. Nematol. 39:133-144.

Stoeckius, M., Maaskola, J., Colombo, T., Rahn, H. P., Friedlander, M. R., Li, N., Chen, W., Piano, F., and Rajewsky, N. 2009. Large-scale sorting of C. elegans embryos reveals the dynamics of small RNA expression. Nat. Methods 6: 745-751.

Turner, S. J., and Evans, K. 1998. The origins, global distribution and biology of potato cyst nematodes (Globodera rostochiensis (Woll.) and Globodera pallida (Stone). Pages 7-26 in: Potato Cyst Nematodes. Biology, Distribution and Control. R. Marks and B. Brodie, eds. University Press, Cambridge, UK.

Tylka, G. L., Niblack, T. L., Walk, T. C., Harkins, K. R., Barnett, L., and Baker, N. K. 1993. Flow cytometric analysis and sorting of Heterodera glycines eggs. J. Nematol. 25:596-602.

van Bezooijen, J. 2006. Methods and Techniques for Nematology. Wageningen University, Wageningen, The Netherlands.

van den Elsen, S., Ave, M., Schoenmakers, N., Landeweert, R., Bakker, J., and Helder, J. 2012. A rapid, sensitive and cost-efficient assay to estimate viability of potato cyst nematodes. Phytopathology 102:140-146. 